\title{
Two examples related to conical energies
}

\author{
DAMIAN DĄBRoWsKI
}

\begin{abstract}
In a recent article (2021) we introduced and studied conical energies. We used them to prove three results: a characterization of rectifiable measures, a characterization of sets with big pieces of Lipschitz graphs, and a sufficient condition for boundedness of nice singular integral operators. In this note we give two examples related to sharpness of these results. One of them is due to Joyce and Mörters (2000), the other is new and could be of independent interest as an example of a relatively ugly set containing big pieces of Lipschitz graphs.
\end{abstract}

\section{Kaksi kartioenergioita koskevaa esimerkkiä}

Tiivistelmä. Viimeaikaisessa työssä (2021) esittelimme ja tutkimme uusia kartioenergioita, ja käytimme niitä kolmen tuloksen todistamiseen: antamaan riittävät ja välttämättömät ehdot toisaalta suoristuville mitoille ja toisaalta Lipschitzin kuvaajien suuria osia sisältäville joukoille sekä antamaan riittävän ehdon siistien singulaaristen integraalioperaattoreiden rajoittuneisuudelle. Tässä tutkimuksessa esitämme kaksi näiden tulosten tarkkuuteen liittyvää esimerkkiä. Yksi näistä on peräisin Joycelta ja Mörtersiltä (2000), mutta toinen on uusi ja kenties sellaisenaan mielenkiintoinen esimerkkinä suhteellisen rumasta joukosta, joka kuitenkin sisältää Lipschitzin kuvaajien suuria osia.

\section{Introduction}

In [Dąb21] we introduced conical energies. Let us recall their definition. Given $x \in \mathbb{R}^{d}, \alpha \in(0, \pi / 2)$ and an $m$-dimensional plane $V \in G(d, m)$, set

$$
K(x, V, \alpha)=\left\{y \in \mathbb{R}^{d}: \operatorname{dist}(y, V+x)<\sin (\alpha)|y-x|\right\} .
$$

In other words, $K(x, V, \alpha)$ denotes the open cone centered at $x$ with direction $V$ and aperture $\alpha .{ }^{*}$ The truncated cone $K(x, V, \alpha) \cap B(x, r)$ will be denoted by $K(x, V, \alpha, r)$.

Definition 1.1. Suppose $\mu$ is a Radon measure on $\mathbb{R}^{d}$, and $x \in \operatorname{supp} \mu$. Let $V \in G(d, d-n), \alpha \in(0, \pi / 2), 1 \leq p<\infty$ and $R>0$. We define the $(V, \alpha, p)$-conical energy of $\mu$ at $x$ up to scale $R$ as

$$
\mathcal{E}_{\mu, p}(x, V, \alpha, R)=\int_{0}^{R}\left(\frac{\mu(K(x, V, \alpha, r))}{r^{n}}\right)^{p} \frac{d r}{r} .
$$

For $E \subset \mathbb{R}^{d}$ we set also $\mathcal{E}_{E, p}(x, V, \alpha, R)=\mathcal{E}_{\left.\mathcal{H}^{n}\right|_{E}, p}(x, V, \alpha, R)$.

Note that the definition above depends on the dimension parameter $n$, so to be more precise one could say that $\mathcal{E}_{\mu, p}(x, V, \alpha, R)$ is the $n$-dimensional $(V, \alpha, p)$-conical energy. For the sake of simplicity, for the rest of the introduction we will consider $n$

https://doi.org/10.54330/afm.113378

2020 Mathematics Subject Classification: Primary 28A75; Secondary 28A78, 42B20.

Key words: Cone, conical density, quantitative rectifiability, big pieces of Lipschitz graphs, singular integral operators.

*In [Dąb21] we defined cones slightly differently, with " $\sin (\alpha)$ " replaced by " $\alpha$ ". Obviously one can pass between the two definitions easily, and the current definition is more natural in $\mathbb{R}^{2}$.

(c) 2022 The Finnish Mathematical Society 
to be fixed, and we will not point out this dependence. The same applies to other definitions. Throughout the paper we will actually work with $n=1$.

For $p=1$ the conical energies were first considered in [CT20] where the authors used them to prove an inequality involving analytic capacity and projections. For $p>1$ they were defined in [Dąb21]. A related quantity was also independently introduced in [BN21] — Badger and Naples used their conical defect to characterize measures concentrated on a countable union of Lipschitz graphs.

In [Dąb21] we used the conical energies to prove three results: a characterization of rectifiable measures, a characterization of sets with big pieces of Lipschitz graphs, and a sufficient condition for boundedness of nice singular integral operators. Below we briefly describe the last two theorems. The aim of this note is to give two examples related to sharpness of these results. For more information on conical energies, as well as a full presentation of results obtained in [Dąb21], we refer the reader to the original paper.

1.1. Big pieces of Lipschitz graphs. We begin by recalling some definitions.

Definition 1.2. We say that a Radon measure $\mu$ is $n$-Ahlfors-David regular (abbreviated as $n$-ADR) if there exists a constant $C>0$ such that for all $x \in E$ and $0<r<\operatorname{diam}(E)$

$$
C^{-1} r^{n} \leq \mu(B(x, r)) \leq C r^{n} .
$$

The constant $C$ will be referred to as the ADR constant of $\mu$. Furthermore, we say that an $\mathcal{H}^{n}$-measurable set $E$ is $n$-ADR if $\left.\mathcal{H}^{n}\right|_{E}$ is $n$-ADR.

Definition 1.3. We say that an $n$-ADR set $E \subset \mathbb{R}^{d}$ has big pieces of Lipschitz graphs (BPLG) if there exist constants $\kappa, L>0$, such that the following holds.

For all balls $B$ centered at $E, 0<r(B)<\operatorname{diam}(E)$, there exists an $n$-dimensional Lipschitz graph $\Gamma$ with $\operatorname{Lip}(\Gamma) \leq L$, such that

$$
\mathcal{H}^{n}(E \cap B \cap \Gamma) \geq \kappa r(B)^{n} .
$$

Sets with BPLG were studied in [DS91, DS93a] as a potential quantitative counterpart of rectifiability. Few characterizations of such sets are available. In [DS93b] they were characterised in terms of the big projections property and the weak geometric lemma, in [MO18] — using $L^{2}$ norms of projections, and very recently in [Orp21] using the plenty of big projections property. In [Dąb21] we characterize the sets with BPLG using the conical energy. More precisely, we show that containing BPLG is equivalent to the following property.

Definition 1.4. Let $1 \leq p<\infty$. We say that a measure $\mu$ has big pieces of bounded energy for $p$, abbreviated as $\operatorname{BPBE}(p)$, if there exist constants $\alpha, \kappa, M_{0}>0$ such that the following holds.

For all balls $B$ centered at $\operatorname{supp} \mu, 0<r(B)<\operatorname{diam}(\operatorname{supp} \mu)$, there exist a set $G_{B} \subset B$ with $\mu\left(G_{B}\right) \geq \kappa \mu(B)$, and a direction $V_{B} \in G(d, d-n)$, such that for all $x \in G_{B}$

$$
\mathcal{E}_{\mu, p}\left(x, V_{B}, \alpha, r(B)\right)=\int_{0}^{r(B)}\left(\frac{\mu\left(K\left(x, V_{B}, \alpha, r\right)\right)}{r^{n}}\right)^{p} \frac{d r}{r} \leq M_{0} .
$$

Theorem 1.5. [Dąb21, Theorem 1.11] Let $1 \leq p<\infty$. Suppose $E \subset \mathbb{R}^{d}$ is $n$-ADR. Then $E$ has BPLG if and only if $\left.\mathcal{H}^{n}\right|_{E}$ has $B P B E(p)$.

It seemed to us rather natural to consider also the following property. 
Definition 1.6. Let $1 \leq p<\infty$. We say that a measure $\mu$ has bounded mean energy for $p(\operatorname{BME}(p))$ if there exist constants $\alpha, M_{0}>0$, and for every $x \in \operatorname{supp} \mu$ there exists a direction $V_{x} \in G(d, d-n)$, such that the following holds.

For all balls $B$ centered at $\operatorname{supp} \mu, 0<r(B)<\operatorname{diam}(\operatorname{supp} \mu)$, we have

$$
\begin{aligned}
\int_{B} \mathcal{E}_{\mu, p}\left(x, V_{x}, \alpha, r(B)\right) d \mu(x) & =\int_{B} \int_{0}^{r(B)}\left(\frac{\mu\left(K\left(x, V_{x}, \alpha, r\right)\right)}{r^{n}}\right)^{p} \frac{d r}{r} d \mu(x) \\
& \leq M_{0} \mu(B) .
\end{aligned}
$$

In other words we require $\mu\left(K\left(x, V_{x}, \alpha, r\right)\right)^{p} r^{-n p} \frac{d r}{r} d \mu(x)$ to be a Carleson measure. This condition looks quite natural due to many similar characterizations of so-called uniformly rectifiable sets, e.g. the geometric lemma of [DS91, DS93a] or the results from [Tol09, Tol12]. In this paper we won't need the definition of uniform rectifiability, but let us note that all sets with BPLG are uniformly rectifiable, and that the BPLG condition is strictly stronger than uniform rectifiability.

It is easy to show, using the compactness of $G(d, d-n)$ and Chebyshev's inequality, that $\operatorname{BME}(p)$ implies $\operatorname{BPBE}(p)$. However, the reverse implication does not hold. In Section 2 we construct the appropriate example.

Theorem 1.7. There exists a $1-A D R$ set $E \subset \mathbb{R}^{2}$ that contains big pieces of 1-Lipschitz graphs, but it does not satisfy the $B M E(p)$ condition for any $p \geq 1$.

The problem with BME is the following. Contrary to the aforementioned characterizations of uniform rectifiability, in BME the "approximating" plane $V_{x}$ is fixed for every $x \in \operatorname{supp} \mu$ once and for all, and we do not allow it to change between different scales. As shown by our example, this is too rigid.

Question 1.8. Suppose one modifies the definition of BME, allowing the planes $V_{x}$ to depend on $r$, perhaps with some additional control on the oscillation of $V_{x, r}$. Can the modified BME be used to characterize BPLG, or uniform rectifiability?

1.2. Boundedness of SIOs. We consider singular integral operators of convolution type, with odd $C^{2}$ kernels $k: \mathbb{R}^{d} \backslash\{0\} \rightarrow \mathbb{R}$ satisfying for some constant $C_{k}>0$

$$
\left|\nabla^{j} k(x)\right| \leq \frac{C_{k}}{|x|^{n+j}} \quad \text { for } x \neq 0 \quad \text { and } \quad j \in\{0,1,2\}
$$

We will denote the class of all such kernels by $\mathcal{K}^{n}\left(\mathbb{R}^{d}\right)$. Note that these kernels are particularly nice examples of Calderón-Zygmund kernels.

Definition 1.9. Given a kernel $k \in \mathcal{K}^{n}\left(\mathbb{R}^{d}\right)$, a constant $\varepsilon>0$, a Radon measure $\mu$ and a function $f \in L_{\text {loc }}^{1}(\mu)$ we define

$$
T_{\mu, \varepsilon} f(x)=\int_{|x-y|>\varepsilon} k(y-x) f(y) d \mu(y), \quad x \in \mathbb{R}^{d} .
$$

We say that $T_{\mu}$ is bounded in $L^{2}(\mu)$ if all $T_{\mu, \varepsilon}$ are bounded in $L^{2}(\mu)$, uniformly in $\varepsilon>0$.

In their seminal work [DS91] David and Semmes showed that, for an $n$-ADR set, the $L^{2}$ boundedness of all singular integral operators with smooth and odd kernels is equivalent to uniform rectifiability. Later on Tolsa [Tol09] improved on this by showing that uniform rectifiability is equivalent to the $L^{2}$ boundedness of all SIOs with kernels in $\mathcal{K}^{n}\left(\mathbb{R}^{d}\right)$. 
The situation in the non-ADR setting is less clear. A necessary condition for the boundedness of SIOs in $L^{2}(\mu)$, where $\mu$ is Radon and non-atomic, is the polynomial growth condition:

$$
\mu(B(x, r)) \leq C_{1} r^{n},
$$

see [Dav91, Proposition 1.4 in Part III]. Some sufficient conditions for boundedness of nice SIOs have been shown in [AT15] and [GS19]. In [Dąb21] we showed that $\operatorname{BPBE}(2)$ is another sufficient condition.

Theorem 1.10. [Dąb21, Theorem 1.17] Let $\mu$ be a Radon measure on $\mathbb{R}^{d}$ satisfying the polynomial growth condition (1.3). Suppose that $\mu$ has BPBE(2). Then, all singular integral operators $T_{\mu}$ with kernels $k \in \mathcal{K}^{n}\left(\mathbb{R}^{d}\right)$ are bounded in $L^{2}(\mu)$, with norm depending only on $\alpha, C_{1}, M_{0}, \kappa$, and the constant $C_{k}$ from (1.2).

The result was inspired by [CT20, Theorem 10.2] where Chang and Tolsa showed an analogous result with $\operatorname{BPBE}(2)$ replaced by $\operatorname{BPBE}(1)$. It is easy to see that for measures satisfying the polynomial growth condition (1.3) we have

$$
\mathcal{E}_{\mu, 2}(x, V, \alpha, R) \leq C_{1} \mathcal{E}_{\mu, 1}(x, V, \alpha, R),
$$

so that $\mathrm{BPBE}(1)$ implies $\mathrm{BPBE}(2)$. In Section 3 we show that the measure constructed in [JM00] does not satisfy the stronger condition of [CT20, Theorem 10.2], but it trivially satisfies the assumptions of Theorem 1.10.

Theorem 1.11. The measure constructed in [JM00] satisfies $B P B E(2)$, but does not satisfy $B P B E(1)$.

Hence, Theorem 1.10 really improves on [CT20, Theorem 10.2]. This also illustrates the following curious phenomenon: in the case of $\operatorname{ADR}$ measures, $\operatorname{BPBE}(p)$ is equivalent to $\operatorname{BPBE}(q)$ for any $p, q \geq 1$, but once we drop the $\mathrm{AD}$ regularity assumption, the conditions are no longer equivalent. In the polynomial growth case (1.3) we only have

if $p \leq q$.

$$
\operatorname{BPBE}(p) \Longrightarrow \operatorname{BPBE}(q)
$$

Finally, let us mention that Theorem 1.10 is sharp in the sense that one cannot replace $\operatorname{BPBE}(2)$ with $\operatorname{BPBE}(p)$ for any $p>2$, see [Dąb21, Remark 1.20]. At the same time, as noted just below [Dąb21, Remark 1.20], BPBE(2) is not a necessary condition for boundedness of nice SIOs.

Acknowledgements. I would like to thank Xavier Tolsa for many helpful conversations, and for his feedback on the first draft of this article.

I received support from the Spanish Ministry of Economy and Competitiveness, through the María de Maeztu Programme for Units of Excellence in R\&D (MDM2014-0445), and also partial support from the Catalan Agency for Management of University and Research Grants (2017-SGR-0395), and from the Spanish Ministry of Science, Innovation and Universities (MTM-2016-77635-P).

Notation. We will write $f \lesssim g$ to denote $f \leq C g$ for some absolute constant $C>0$. If $C$ depends on parameter $t$, we will write $f \lesssim_{t} g$. Moreover, $f \approx g$ denotes $f \lesssim g \lesssim f$

Throughout the article we will be only working with cones in $\mathbb{R}^{2}$, and so it will be convenient to use the following notation: given $\theta \in[0, \pi)$ let $V_{\theta} \in G(2,1)$ be the line forming angle $\theta$ with the $x$-axis, i.e.

$$
V_{\theta}=\{(x, \tan (\theta) x): x \in \mathbb{R}\}
$$


for $\theta \neq \pi / 2$, and $V_{\pi / 2}$ the vertical axis. We will write

$$
K(x, \theta, \alpha):=K\left(x, V_{\theta}, \alpha\right),
$$

and similarly $K(x, \theta, \alpha, r):=K\left(x, V_{\theta}, \alpha, r\right)$.

Given two lines $V_{1}, V_{2}$, we denote by $\measuredangle\left(V_{1}, V_{2}\right) \in[0, \pi / 2]$ the angle between $V_{1}$ and $V_{2}$.

\section{Set with BPLG but no BME}

We will show the following.

Proposition 2.1. Fix an aperture parameter $\alpha \in(0, \pi / 2)$. There exists a sequence of $1-A D R$ sets $E_{N}=E_{N}(\alpha) \subset B(0,1) \subset \mathbb{R}^{2}, N \geq 100\left(1+\log _{2}\left(\alpha^{-1}\right)\right)$, with the following properties:

(1) they all contain BPLG in a uniform way, that is, they are 1-ADR with some absolute constant $C$, and they all satisfy the BPLG condition with $L=1$ and some absolute constant $\kappa>0$.

(2) regardless of the choice of directions $\theta_{x} \in[0, \pi)$, for all $p \geq 1$ they have big conical energies:

$$
\begin{aligned}
& \int_{E_{N}} \mathcal{E}_{E_{N}, p}\left(x, \theta_{x}, \alpha, 1\right) d \mathcal{H}^{1}(x) \\
& =\int_{E_{N}} \int_{0}^{1}\left(\frac{\mathcal{H}^{1}\left(K\left(x, \theta_{x}, \alpha, r\right) \cap E_{N}\right)}{r}\right)^{p} \frac{d r}{r} d \mathcal{H}^{1}(x) \gtrsim_{\alpha} N .
\end{aligned}
$$

For the sake of clarity, we will only prove (2.1) for $p=1$ - the proof for other $p$ is virtually the same. More precisely, to show (2.1) we find a large subset of $E_{N}$ (with length depending on $\alpha$ ) such that for any $x$ in the subset and any direction $\theta$ we have $\mathcal{H}^{1}\left(K(x, \theta, \alpha, r) \cap E_{N}\right) / r \gtrsim 1$ at $\approx N$ distinct dyadic scales $0<r<1$. Thus, $\left(\mathcal{H}^{1}\left(K(x, \theta, \alpha, r) \cap E_{N}\right) / r\right)^{p} \gtrsim 1$ at the same dyadic scales, which gives (2.1) for arbitrary $p \geq 1$. See the proofs of Lemma 2.7 and Lemma 2.11.

Let $\alpha_{k} \rightarrow 0$. Now, a disjoint union of appropriately rescaled sets $E_{N}\left(\alpha_{k}\right)$, with $k, N \rightarrow \infty$, would contain BPLG and would not satisfy the $\operatorname{BME}(p)$ condition (Definition 1.6) for any $M_{0}$ and $\alpha>0$. We omit the details.

Without loss of generality we will assume that $\alpha>0$ is smaller than some absolute constant, which is smaller than $\pi / 100$, say (note that taking smaller $\alpha$ makes (2.1) more difficult to prove). Let $M=100\left\lceil\alpha^{-1}\right\rceil$, so that $M \approx \alpha^{-1}$. In the lemma below we construct a Lipschitz graph $\Gamma=\Gamma(N, M)$ that can be seen as the first approximation of the set $E_{N}$. For all directions $\theta$ in $[0, \pi / 4]$ the conical energy $\mathcal{E}_{\Gamma, 1}(x, V, \alpha, 1)$ is bigger than $N$ for all $x$ belonging to a neighbourhood of a large portion of $\Gamma$. Rescaled and rotated copies of $\Gamma$ will be then used as building blocks in the construction of $E_{N}$.

Let $\Delta$ be the usual dyadic grid of open intervals on $(-1,1)$, and let $\Delta_{k}$ denote the dyadic intervals of length $2^{-k}$.

Lemma 2.2. Let $N \geq 100\left(1+\log _{2}\left(\alpha^{-1}\right)\right)$ be an integer. There exists a piecewise linear 1-Lipschitz function $g:[-1,1] \rightarrow\left[-M^{-1}, M^{-1}\right]$, and a collection of disjoint dyadic intervals $\mathcal{I} \subset \Delta$ with the following properties:

(P1) $g(-1)=g(1)=0$.

(P2) For every $I \in \mathcal{I}$ we have $I \subset[-1 / 2,1 / 2]$, the function $\left.g\right|_{I}$ is increasing, and for $t \in I$ we have $g^{\prime}(t)=1$. 
(P3) $\# \mathcal{I}=2^{-M} 2^{N(M+1)}$ and $\mathcal{I} \subset \Delta_{N(M+1)}$. Hence,

$$
\mathcal{H}^{1}\left(\bigcup_{I \in \mathcal{I}} I\right)=2^{-M} \approx_{\alpha} 1 .
$$

(P4) Let $\Gamma=\operatorname{graph}(g), G:[-1,1] \rightarrow \Gamma$ be the graph map $G(t)=(t, g(t))$ For any $I \in \mathcal{I}$, any $x \in \mathbb{R}^{2}$ with $\operatorname{dist}(x, G(I))<2^{-N(M+1)}$, and all $\theta \in[0, \pi / 4]$, we have

$$
\int_{0}^{1} \frac{\mathcal{H}^{1}(K(x, \theta, \alpha, r) \cap \Gamma)}{r} \frac{d r}{r} \gtrsim N .
$$

For an idea of what $\Gamma$ looks like, see the graph at the bottom of Figure 2.2. Before we prove Lemma 2.2, let us show how it can be used to prove Proposition 2.1.

2.1. Construction of $\boldsymbol{E}_{N}$. Let $\Gamma=\Gamma(M, N)$ be the 1-Lipschitz graph from Lemma 2.2. The set $E_{N}$ will consist of one "big" Lipschitz graph $\Gamma_{0}=\Gamma$, and three layers of much smaller Lipschitz graphs stacked on top of the big one. The small graphs will be rescaled and rotated versions of $\Gamma$. Roughly speaking, the big graph ensures big conical energy in directions $[0, \pi / 4]$, the first layer of small graphs ensures big conical energy in direction $[\pi / 4, \pi / 2]$, and so on.

Another way to see $E_{N}$ is as a union of four bilipschitz curves $\Gamma_{0}, \ldots, \Gamma_{3}$, and this is how we are going to define it. If $\Gamma_{i}$ is already defined, $\Gamma_{i+1}$ will be constructed by replacing some of the segments comprising $\Gamma_{i}$ with rescaled and rotated copies of $\Gamma$.

First, let $\rho: \mathbb{R}^{2} \rightarrow \mathbb{R}^{2}$ be the counterclockwise rotation by $\pi / 4$. Set $L_{0}=$ $\{(x, 0): x \in \mathbb{R}\}$ and for $k \geq 1$ set $L_{k}=\rho^{k}\left(L_{0}\right) \in G(2,1)$ (here $\rho^{k}$ denotes $k$ compositions of $\rho$, and the same notation is used for $\delta$ defined below).

Define also $r_{k}=2^{-k N(M+1)-k / 2}$, and let $\delta: \mathbb{R}^{2} \rightarrow \mathbb{R}^{2}$ be the dilation by factor $r_{1}$, i.e. $\delta(x)=r_{1} x$. Note that $r_{k}=\left(r_{1}\right)^{k}$, so that $\delta^{k}$ is the dilation by factor $r_{k}$. The constant $r_{1}$ was chosen in such a way that for an interval $I \in \mathcal{I} \subset \Delta_{N(M+1)}$ we have $\mathcal{H}^{1}(G(I))=2 r_{1}$ by (P2) (where $G$ is the graph map of $g$ ).

We will abuse the notation and identify the segment $S_{0}:=[-1,1] \times\{0\}$ with $[-1,1] \subset \mathbb{R}$.

Set $\Gamma_{0}=\Gamma$, and let $\gamma_{0}=\sigma_{0}: S_{0} \rightarrow \Gamma_{0}$ be defined as the natural graph map $\gamma_{0}(t)=\sigma_{0}(t)=G(t)=(t, g(t))$.

Lemma 2.3. Let $k \in\{1,2,3\}$. Denote by $\mathcal{I}^{k}$ the $k$-fold Cartesian product of $\mathcal{I}$, where $\mathcal{I}$ is the family of intervals from Lemma 2.2. There exist $\gamma_{k}: S_{0} \rightarrow \mathbb{R}^{2}, \Gamma_{k}:=$ $\gamma_{k}\left(S_{0}\right)$, and for each $I=\left(I_{1}, \ldots, I_{k}\right) \in \mathcal{I}^{k}$ there exist sets $S_{k, I}, \Gamma_{k, I}$, and a map $G_{k, I}: \mathbb{R}^{2} \rightarrow \mathbb{R}^{2}$, such that:

a) $G_{k, I}:=\tau_{I} \circ \rho^{k} \circ \delta^{k}$ for some translation $\tau_{I}$, and $S_{k, I}:=G_{k, I}\left(S_{0}\right)$ are segments (in particular, $\mathcal{H}^{1}\left(S_{k, I}\right)=2 r_{k}$ and $S_{k, I}$ are parallel to $L_{k}$ ),

b) $\Gamma_{k, I}$ are rescaled and rotated copies of $\Gamma$, with $\Gamma_{k, I}:=G_{k, I}\left(\Gamma_{0}\right)$ (in particular, since the endpoints of $\Gamma_{0}$ and $S_{0}$ coincide, the same is true for $\Gamma_{k, I}$ and $S_{k, I}$ ),

c) $\Gamma_{k}:=\gamma_{k}\left(S_{0}\right)$ are of the form

$$
\Gamma_{k}=\left(\Gamma_{k-1} \backslash \bigcup_{I \in \mathcal{I}^{k}} S_{k, I}\right) \cup \bigcup_{I \in \mathcal{I}^{k}} \Gamma_{k, I},
$$

d) for $k=1, J \in \mathcal{I}$, we have $S_{1, J}=\sigma_{0}(J) \subset \Gamma_{0}$, and for $k>1$, if $I=\left(I^{\prime}, J\right) \in$ $\mathcal{I}^{k-1} \times \mathcal{I}$, then $S_{k, I}=G_{k-1, I^{\prime}}\left(S_{1, J}\right) \subset \Gamma_{k-1, I^{\prime}} \subset \Gamma_{k-1}$, 
e) if $I=\left(I^{\prime}, J\right), a_{1}, a_{2}$ are the endpoints of $S_{k, I}$, and $b_{1}, b_{2}$ are the endpoints of $\Gamma_{k-1, I^{\prime}}$, then

$$
\left|a_{i}-b_{j}\right| \gtrsim r_{k-1}
$$

for $i, j \in\{1,2\}$ (i.e. $S_{k, I}$ is "deep inside" $\Gamma_{k-1, I^{\prime}}$ ),

f) the maps $\gamma_{k}$ are of the form $\gamma_{k}=\sigma_{k} \circ \cdots \circ \sigma_{0}$, where $\sigma_{k}: \Gamma_{k-1} \rightarrow \Gamma_{k}$ is defined as

$$
\sigma_{k}(x)= \begin{cases}x, & \text { for } x \in \Gamma_{k-1} \backslash \bigcup_{I \in \mathcal{I}^{k}} S_{k, I}, \\ G_{k, I}(x) \circ \sigma_{0} \circ G_{k, I}^{-1}(x), & \text { for } x \in S_{k, I}, I \in \mathcal{I}^{k} .\end{cases}
$$

In particular, $\sigma_{k}\left(S_{k, I}\right)=\Gamma_{k, I}$.

g) $\left\|\sigma_{k}-i d\right\|_{L^{\infty}\left(\Gamma_{k-1}\right)} \leq 2 M^{-1} r_{k}$,

Proof of Lemma 2.3. We will define $\sigma_{k}$ inductively. First, for any $I \in \mathcal{I}$ set $S_{1, I}:=\sigma_{0}(I) \subset \Gamma_{0}$. Observe that by (P2) $S_{1, I}$ is a segment parallel to $L_{1}$. Moreover, since $\mathcal{H}^{1}(I)=2^{1 / 2} r_{1}$, we have $\mathcal{H}^{1}\left(S_{1, I}\right)=2 r_{1}$. It follows that $S_{1, I}=\tau_{I} \circ \rho \circ \delta\left(S_{0}\right)$ for some translation $\tau_{I}$. Define $G_{1, I}: \mathbb{R}^{2} \rightarrow \mathbb{R}^{2}$ as $G_{1, I}=\tau_{I} \circ \rho \circ \delta$, and $\Gamma_{1, I}=G_{1, I}\left(\Gamma_{0}\right)$.

We define $\sigma_{1}: \Gamma_{0} \rightarrow \mathbb{R}^{2}$ as in $\mathrm{f}$ ). In other words, $\left.\sigma_{1}\right|_{S_{1, I}}$ can be seen as a graph map parametrizing the Lipschitz graph $\Gamma_{1, I}$. It is very easy to see that $S_{1, I}, \Gamma_{1, I}$, and $\sigma_{1}$ defined in this way satisfy all the conditions except for e) and g), which we will prove later on.

Now, suppose that $\sigma_{k-1}, \gamma_{k-1}$, etc. have already been defined, and that they satisfy a)-d), f).

For any $I=\left(I^{\prime}, J\right) \in \mathcal{I}^{k-1} \times \mathcal{I}$ set $S_{k, I}:=G_{k-1, I^{\prime}}\left(S_{1, J}\right) \subset \Gamma_{k-1, I^{\prime}}$. Since $S_{1, J}$ is parallel to $L_{1}$ and $G_{k-1, I^{\prime}}=\tau_{I^{\prime}} \circ \rho^{k-1} \circ \delta^{k-1}, S_{k, I}$ is a segment parallel to $L_{k}$. Moreover, since $\mathcal{H}^{1}\left(S_{1, J}\right)=2 r_{1}$, we have $\mathcal{H}^{1}\left(S_{k, I}\right)=2 r_{1} r_{k-1}=2 r_{k}$. It follows that $S_{k, I}=\tau_{I} \circ \rho^{k} \circ \delta^{k}\left(S_{0}\right)$ for some translation $\tau_{I}$.

We define $\sigma_{k}: \Gamma_{0} \rightarrow \mathbb{R}^{2}$ as in f), so that $\left.\sigma_{k}\right|_{S_{k, I}}$ can be seen as a graph map parametrizing the Lipschitz graph $\Gamma_{k, I}$. It is easy to see that $\sigma_{k}, \Gamma_{k}$, etc. defined this way satisfy a)-d), f).

Proof of e). Let $k=1$. Recall that for all $I \in \mathcal{I}$ we have $I \subset[-1 / 2,1 / 2]$ by (P2). Hence, $S_{1, I}=\sigma_{0}(I) \subset \sigma_{0}([-1 / 2,1 / 2]) \subset \Gamma_{0}$. If $x \in \sigma_{0}([-1 / 2,1 / 2])$ is arbitrary and if $y \in \Gamma_{0}$ is one of the endpoints of $\Gamma_{0}$, we have $|x-y| \gtrsim 1=r_{0}$. So e) holds for $k=1$. For $k \in\{2,3\}$ the claim follows from the fact that if $I=\left(I^{\prime}, J\right) \in \mathcal{I}^{k-1} \times \mathcal{I}$, then $S_{k, I}=G_{k-1, I^{\prime}}\left(S_{1, J}\right)$ and $\Gamma_{k-1, I^{\prime}}=G_{k-1, I^{\prime}}\left(\Gamma_{0}\right)$.

Proof of g). We have $\sigma_{k}=i d$ on $\Gamma_{k-1} \backslash \bigcup_{I \in \mathcal{I}^{k}} S_{k, I}$, and for $x \in S_{k, I}$

$$
\begin{aligned}
\left|\sigma_{k}(x)-x\right| & =\left|G_{k, I} \circ \sigma_{0} \circ G_{k, I}^{-1}(x)-G_{k, I} \circ G_{k, I}^{-1}(x)\right| \\
& =r_{k}\left|\sigma_{0} \circ G_{k, I}^{-1}(x)-G_{k, I}^{-1}(x)\right| \leq r_{k}\|g\|_{\infty} \leq 2 M^{-1} r_{k},
\end{aligned}
$$

where we used the fact that $\sigma_{0}(t)=(t, g(t))$, and that $\|g\|_{\infty} \leq 2 M^{-1}$ by Lemma 2.2.

Lemma 2.4. The maps $\gamma_{k}$ and $\sigma_{k}$ from Lemma 2.3 are bilipschitz, with bilipschitz constants independent of $N$ and $\alpha$.

Proof. It suffices to show that $\sigma_{k}$ is bilipschitz with $\operatorname{Lip}\left(\sigma_{k}\right)$ and $\operatorname{Lip}\left(\sigma_{k}^{-1}\right)$ independent of $N, \alpha$, and then the same will be true for $\gamma_{k}$ by Lemma $2.3 \mathrm{f}$ ).

Suppose that $\sigma_{j}$ are already known to be bilipschitz for $0 \leq j \leq k-1$, with $\operatorname{Lip}\left(\sigma_{j}\right)$ and $\operatorname{Lip}\left(\sigma_{j}^{-1}\right)$ independent of $N, \alpha$ (clearly, the condition holds for $\sigma_{0}$ ). Let $x, y \in \Gamma_{k-1}$. Our aim is to show that $\left|\sigma_{k}(x)-\sigma_{k}(y)\right| \approx|x-y|$. 
Case 1. $|x-y|>6 M^{-1} r_{k}$. It follows from Lemma $\left.2.3 \mathrm{~g}\right)$ that $\left|\sigma_{k}(x)-\sigma_{k}(y)\right| \leq|x-y|+\left|\sigma_{k}(x)-x\right|+\left|\sigma_{k}(y)-y\right| \leq|x-y|+4 M^{-1} r_{k} \leq 2|x-y|$, and $\left|\sigma_{k}(x)-\sigma_{k}(y)\right| \geq|x-y|-\left|\sigma_{k}(x)-x\right|-\left|\sigma_{k}(y)-y\right| \geq|x-y|-4 M^{-1} r_{k} \geq \frac{1}{3}|x-y|$.

Case 2. $x, y \in \Gamma_{k-1} \backslash \bigcup_{I \in \mathcal{I}^{k}} S_{k, I}$. This case is trivial, because $\left|\sigma_{k}(x)-\sigma_{k}(y)\right|=$ $|x-y|$.

Case 3. $|x-y| \leq 6 M^{-1} r_{k}$, and $x, y \in \overline{S_{k, I}}$ for some $I \in \mathcal{I}^{k}$. Using the fact that $\sigma_{0}$ is bilipschitz we get

$$
\begin{aligned}
\left|\sigma_{k}(x)-\sigma_{k}(y)\right| & =\left|G_{k, I} \circ \sigma_{0} \circ G_{k, I}^{-1}(x)-G_{k, I} \circ \sigma_{0} \circ G_{k, I}^{-1}(y)\right| \\
& =r_{k}\left|\sigma_{0} \circ G_{k, I}^{-1}(x)-\sigma_{0} \circ G_{k, I}^{-1}(y)\right| \approx r_{k}\left|G_{k, I}^{-1}(x)-G_{k, I}^{-1}(y)\right|=|x-y| .
\end{aligned}
$$

Case 4. $|x-y| \leq 6 M^{-1} r_{k}, x \in S_{k, I}$ for some $I \in \mathcal{I}^{k}$, and $y \in \Gamma_{k-1} \backslash \overline{S_{k, I}}$. We claim that

$$
y \in \Gamma_{k-1, I^{\prime}}
$$

where $I=\left(I^{\prime}, J\right) \in \mathcal{I}^{k-1} \times \mathcal{I}$ and $\Gamma_{k-1, I^{\prime}}$ is the Lipschitz graph containing $S_{k, I}$. Indeed, by the induction assumption, the map $\gamma_{k-1}^{-1}: \Gamma_{k-1} \rightarrow S_{0}$ is bilipschitz with $\operatorname{Lip}\left(\gamma_{k-1}\right), \operatorname{Lip}\left(\gamma_{k-1}^{-1}\right)$ independent of $N, \alpha$. Since $\mathcal{H}^{1}\left(S_{k, I}\right)=2 r_{k}$ and $\mathcal{H}^{1}\left(\Gamma_{k-1, I^{\prime}}\right) \approx$ $r_{k-1}$, we get that

$$
\mathcal{H}^{1}\left(\gamma_{k-1}^{-1}\left(S_{k, I}\right)\right) \approx r_{k} \quad \text { and } \quad \mathcal{H}^{1}\left(\gamma_{k-1}^{-1}\left(\Gamma_{k-1, I^{\prime}}\right)\right) \approx r_{k-1} .
$$

Moreover, we have

$$
\gamma_{k-1}^{-1}\left(S_{k, I}\right) \subset \gamma_{k-1}^{-1}\left(\Gamma_{k-1, I^{\prime}}\right) \subset S_{0},
$$

where all three sets are segments. If $a_{1}, a_{2}$ and $b_{1}, b_{2}$ are the endpoints of $\gamma_{k-1}^{-1}\left(S_{k, I}\right)$ and $\gamma_{k-1}^{-1}\left(\Gamma_{k-1, I^{\prime}}\right)$, respectively, then it follows from Lemma $\left.2.3 \mathrm{e}\right)$ and from the bilipschitz property of $\gamma_{k-1}$ that for $i, j \in\{1,2\}$ we have

$$
\left|a_{i}-b_{j}\right| \gtrsim r_{k-1} \text {. }
$$

Recall that $x \in S_{k, I}$ and $|x-y| \lesssim M^{-1} r_{k}$, so that $\operatorname{dist}\left(y, S_{k, I}\right) \lesssim M^{-1} r_{k}$. Hence,

$$
\operatorname{dist}\left(\gamma_{k-1}^{-1}(y), \gamma_{k-1}^{-1}\left(S_{k, I}\right)\right) \lesssim M^{-1} r_{k} .
$$

Putting this together with (2.4) and (2.5), and assuming that $M \geq M_{0}$ for some absolute constant $M_{0}>10$, we get that $\gamma_{k-1}^{-1}(y) \in \gamma_{k-1}^{-1}\left(\Gamma_{k-1, I^{\prime}}\right)$, which is equivalent to $y \in \Gamma_{k-1, I^{\prime}}$.

Now, let $z \in \overline{S_{k, I}}$ be an endpoint of $S_{k, I}$ minimizing the distance to $x$. Observe that $x-z \in L_{k}$ and $\sigma_{k}(x)-x \in L_{k}^{\perp}$, so

$$
\left|\sigma_{k}(x)-z\right|^{2}=\left|\sigma_{k}(x)-x\right|^{2}+|x-z|^{2} .
$$

Moreover, since $z$ is an endpoint of $S_{k, I}$, the point $G_{k, I}^{-1}(z)$ is an endpoint of $S_{0}$, and so by $(\mathrm{P} 1) g\left(G_{k, I}^{-1}(z)\right)=0$. Together with the fact that $g$ is 1-Lipschitz this gives

$$
\begin{aligned}
\left|\sigma_{k}(x)-x\right| & =\left|G_{k, I} \circ \sigma_{0} \circ G_{k, I}^{-1}(x)-G_{k, I} \circ G_{k, I}^{-1}(x)\right| \\
& =r_{k}\left|\sigma_{0} \circ G_{k, I}^{-1}(x)-G_{k, I}^{-1}(x)\right|=r_{k}\left|g\left(G_{k, I}^{-1}(x)\right)\right| \\
& =r_{k}\left|g\left(G_{k, I}^{-1}(x)\right)-g\left(G_{k, I}^{-1}(z)\right)\right| \leq r_{k}\left|G_{k, I}^{-1}(x)-G_{k, I}^{-1}(z)\right|=|x-z| .
\end{aligned}
$$


Furthermore, observe that since $y \in \Gamma_{k-1, I^{\prime}} \backslash \overline{S_{k, I}}, z \in \overline{S_{k, I}}$ is an endpoint of $S_{k, I}$, $\mathcal{H}^{1}\left(S_{k, I}\right)=2 r_{k}$, and $|x-y| \lesssim M^{-1} r_{k}$, we get that the point $\gamma_{k-1}^{-1}(z) \in S_{0}$ lies between the points $\gamma_{k-1}^{-1}(x)$ and $\gamma_{k-1}^{-1}(y)$. We already know that $\gamma_{k-1}$ is bilipschitz, and so

$$
\begin{aligned}
|x-z|+|z-y| & \approx\left|\gamma_{k-1}^{-1}(x)-\gamma_{k-1}^{-1}(z)\right|+\left|\gamma_{k-1}^{-1}(z)-\gamma_{k-1}^{-1}(y)\right| \\
& =\left|\gamma_{k-1}^{-1}(x)-\gamma_{k-1}^{-1}(y)\right| \approx|x-y| .
\end{aligned}
$$

Now, we need to further differentiate between two subcases.

Subcase 4a. $|x-y| \leq 6 M^{-1} r_{k}, x \in S_{k, I}$, and $y \in S_{k, Y}$ for some $Y \in \mathcal{I}^{k}, I \neq Y$. We claim that the point $z$ is a common endpoint of $S_{k, Y}$ and $S_{k, I}$. Indeed, since $y \in \Gamma_{k-1, I^{\prime}}$ by $(2.3)$, we have $Y=\left(I^{\prime}, Z\right) \in \mathcal{I}^{k-1} \times \mathcal{I}$ and $S_{k, Y} \subset \Gamma_{k-1, I^{\prime}}$. By Lemma $2.3 \mathrm{~d}) S_{k, Y}=G_{k-1, I^{\prime}}\left(S_{1, Z}\right)=G_{k-1, I^{\prime}} \circ \sigma_{0}(Z)$, and $S_{k, I}=G_{k-1, I^{\prime}} \circ \sigma_{0}(J)$. Recall that $|x-y| \leq 6 M^{-1} r_{k}$, which implies $\operatorname{dist}\left(S_{k, I}, S_{k, Y}\right) \leq 6 M^{-1} r_{k}$, and so $\operatorname{dist}(Z, J) \lesssim M^{-1} r_{k-1}^{-1} r_{k}=M^{-1} r_{1}$. By (P3) $J$ and $Z$ are dyadic intervals of length $\sqrt{2} r_{1}$, which implies that $\operatorname{dist}(Z, J)=0$. Hence, the point $z$ is a common endpoint of $S_{k, I}$ and $S_{k, Y}$, and the estimates (2.6), (2.7) are also valid with $x$ replaced by $y$.

The Lipschitz property of $\sigma_{k}$ follows easily:

$$
\left|\sigma_{k}(x)-\sigma_{k}(y)\right| \leq\left|\sigma_{k}(x)-z\right|+\left|z-\sigma_{k}(y)\right| \stackrel{(2.6),(2.7)}{\lesssim}|x-z|+|z-y| \stackrel{(2.8)}{\approx}|x-y| .
$$

The converse inequality is a consequence of the fact that $S_{k, I}$ and $S_{k, Y}$ are co-linear, $x-y \in L_{k}, \sigma_{k}(x)-x \in L_{k}^{\perp}$, and $\sigma_{k}(y)-y \in L_{k}^{\perp}$ :

$$
\begin{aligned}
\left|\sigma_{k}(x)-\sigma_{k}(y)\right|^{2} & =\left|\sigma_{k}(x)-x+x-y+y-\sigma_{k}(y)\right|^{2} \\
& =|x-y|^{2}+\left|\sigma_{k}(x)-x+y-\sigma_{k}(y)\right|^{2} \geq|x-y|^{2} .
\end{aligned}
$$

Subcase 4b. $|x-y| \leq 6 M^{-1} r_{k}, x \in S_{k, I}$ for some $I \in \mathcal{I}^{k}$, and $y \in \Gamma_{k-1} \backslash$ $\bigcup_{Y \in \mathcal{I}^{k}} S_{k, Y}$. In this case we have $\sigma_{k}(y)=y$. The upper bound follows from previous estimates:

$$
\left|\sigma_{k}(x)-y\right| \leq\left|\sigma_{k}(x)-z\right|+|z-y| \stackrel{(2.6),(2.7)}{\lesssim}|x-z|+|z-y| \stackrel{(2.8)}{\approx}|x-y| .
$$

Concerning the lower bound, it follows by elementary geometry and properties of our construction that $\pi / 4 \leq \measuredangle\left(\sigma_{k}(x), z, y\right) \leq \pi$, see Figure 2.1. Thus, using the law of cosines

$$
\begin{aligned}
\left|\sigma_{k}(x)-y\right|^{2} & =\left|\sigma_{k}(x)-z\right|^{2}+|z-y|^{2}-2\left|\sigma_{k}(x)-z\right||z-y| \cos \left(\measuredangle\left(\sigma_{k}(x), z, y\right)\right) \\
& \geq\left|\sigma_{k}(x)-z\right|^{2}+|z-y|^{2}-\sqrt{2}\left|\sigma_{k}(x)-z\right||z-y| \\
& \geq\left(1-\frac{\sqrt{2}}{2}\right)\left(\left|\sigma_{k}(x)-z\right|^{2}+|z-y|^{2}\right) \stackrel{(2.6)}{\geq}|x-z|^{2}+|z-y|^{2} \gtrsim|x-y|^{2} .
\end{aligned}
$$

Since this was the last case we had to check, we get that $\sigma_{k}$ is bilipschitz, as claimed.

Finally, we set

$$
E_{N}=\Gamma_{0} \cup \Gamma_{1} \cup \Gamma_{2} \cup \Gamma_{3} .
$$

Note that due to Lemma $2.3 \mathrm{c}$ )

$$
E_{N}=\Gamma_{0} \cup \bigcup_{I \in \mathcal{I}} \Gamma_{1, I} \cup \bigcup_{I \in \mathcal{I}^{2}} \Gamma_{2, I} \cup \bigcup_{I \in \mathcal{I}^{3}} \Gamma_{3, I}
$$

That is, $E_{N}$ is a union of a single big Lipschitz graph, and three layers of smaller Lipschitz graphs. 


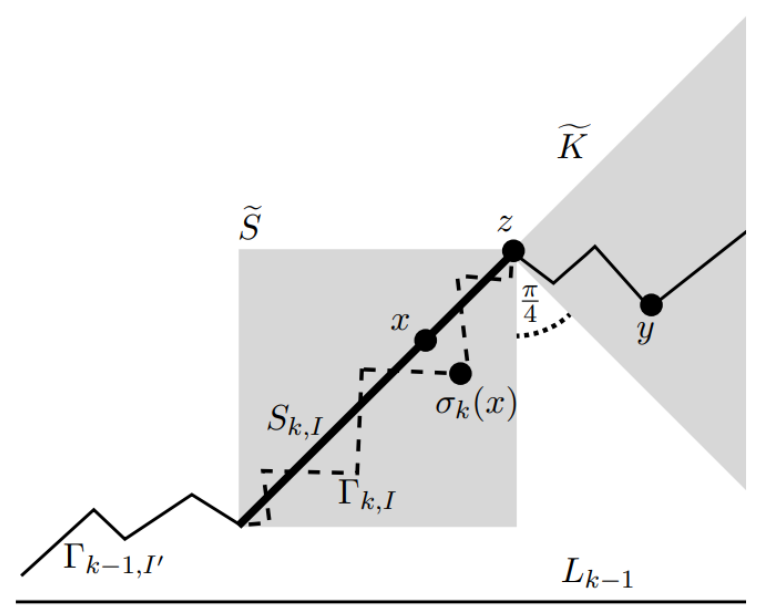

Figure 2.1. Points $x, y, z$ lie on $\Gamma_{k-1, I^{\prime}}$ (continuous curve above), which is a 1-Lipschitz graph over the line $L_{k-1} . x$ belongs to the segment $S_{k, I} \subset \Gamma_{k-1, I^{\prime}}$ (thick segment above), and $z$ is an endpoint of $S_{k, I} . \sigma_{k}(x)$ lies on $\Gamma_{k, I}$ (dashed curve above), a 1-Lipschitz graph over $S_{k, I}$ with the same endpoints as $S_{k, I}$. The 1-Lipschitz property implies that $\Gamma_{k, I} \subset \widetilde{S}$, where $\widetilde{S}$ is a square having $S_{k, I}$ as diagonal. On the other hand, the 1-Lipschitz property of $\Gamma_{k-1, I^{\prime}}$ implies that $\Gamma_{k-1, I^{\prime}} \subset$ $K_{0}:=\overline{K\left(z, L_{k-1}, \pi / 4\right)}$, i.e. it lies in the two-sided version of cone $\widetilde{K}$ above. In particular, $y \in K_{0}$. However, in Subcase $4 \mathrm{~b}$ we assume that $|x-y| \leq 6 M^{-1} r_{k}$ and $y \notin S_{k, I}$, and so $y$ must lie in $\widetilde{K}$, and not in the other one-sided cone comprising $K_{0}$. Since $L_{k-1}$ and $S_{k, I}$ form an angle $\pi / 4$, the observations above imply $\pi / 4 \leq \measuredangle\left(\sigma_{k}(x), z, y\right) \leq \pi$ (see the dotted angle).

2.2. $\boldsymbol{E}_{N}$ has BPLG. In this section we show that $E_{N}$ has big pieces of Lipschitz graphs, with constants independent of $N$. Observe that $E_{N}$ is AD-regular because it is a union of four bilipschitz curves. The ADR constants do not depend on $N$ due to Lemma 2.4.

Lemma 2.5. For any $x \in E_{N}$ and any $0<r<\operatorname{diam}\left(E_{N}\right)$ we can find a Lipschitz graph $\Sigma$ (depending on $x$ and $r$ ) such that

$$
\mathcal{H}^{1}\left(E_{N} \cap B(x, r) \cap \Sigma\right) \gtrsim r
$$

with the implicit constant independent of $N, \alpha$.

First, we prove an auxiliary estimate. Given integers $i, l \in\{0,1,2,3\}$ define $\gamma_{l, i}: \Gamma_{l} \rightarrow \Gamma_{i}$ as $\gamma_{l, i}=\gamma_{i} \circ \gamma_{l}^{-1}$.

Lemma 2.6. Let $i, l \in\{0,1,2,3\}$ and $k=\min (i, l)$. Then

$$
\left\|\gamma_{l, i}-i d\right\|_{L^{\infty}\left(\Gamma_{l}\right)} \leq 6 M^{-1} r_{k+1} .
$$

Proof. If $i=l$ the result is clear because $\gamma_{l, i}=i d$. Assume $l>i$. Applying $(l-i)$-many times Lemma $2.3 \mathrm{~g})$ we get that

$$
\begin{aligned}
\left|x-\gamma_{l, i}(x)\right| & \leq \sum_{j=i+1}^{l}\left|\gamma_{l, j-1}(x)-\gamma_{l, j}(x)\right|=\sum_{j=i+1}^{l}\left|\gamma_{l, j-1}(x)-\sigma_{j}\left(\gamma_{l, j-1}(x)\right)\right| \\
& \leq \sum_{j=i+1}^{l} 2 M^{-1} r_{j} \leq 2(j-k) M^{-1} r_{i+1} \leq 6 M^{-1} r_{i+1} .
\end{aligned}
$$

On the other hand, if $l<i$, then applying the estimate above to $y=\gamma_{l, i}(x)$ we get

$$
\left|x-\gamma_{l, i}(x)\right|=\left|\gamma_{i, l}(y)-y\right| \leq 6 M^{-1} r_{l+1} .
$$


Proof of Lemma 2.5. Let $x \in E_{N}$ and $0<r<\operatorname{diam}\left(E_{N}\right)$. By (2.9) there exist $j \in\{0,1,2,3\}$ and $I \in \mathcal{I}^{j}$ such that $x \in \Gamma_{j, I}$. Suppose $r<r_{j}$. Since $\Gamma_{j, I}$ is a Lipschitz graph satisfying $\mathcal{H}^{1}\left(\Gamma_{j, I}\right) \geq r_{j}>r$, we have

$$
\mathcal{H}^{1}\left(E_{N} \cap B(x, r) \cap \Gamma_{j, I}\right)=\mathcal{H}^{1}\left(B(x, r) \cap \Gamma_{j, I}\right) \gtrsim r .
$$

That is, we may choose $\Sigma=\Gamma_{j, I}$.

Now assume $r_{j} \leq r<r_{0}=1$. Let $k \in\{0,1,2\}$ be such that $r_{k+1} \leq r<r_{k}$ (of course, $k+1 \leq j)$. Let $y=\gamma_{j, k}(x)$. Observe that, by Lemma $\left.2.3 \mathrm{c}\right)$, since $y \in \Gamma_{k}$, there exists some $k^{\prime} \in\{0, \ldots, k\}$ such that $y \in \Gamma_{k^{\prime}, I^{\prime}}$ for some $I^{\prime} \in \mathcal{I}^{k^{\prime}}$. Since $k^{\prime} \leq k$, we have $\mathcal{H}^{1}\left(\Gamma_{k^{\prime}, I^{\prime}}\right) \approx r_{k^{\prime}} \geq r_{k}>r$. Moreover, assuming $M \geq 12$, (2.11) gives

$$
\operatorname{dist}\left(x, \Gamma_{k^{\prime}, I^{\prime}}\right) \leq|x-y|=\left|x-\gamma_{j, k}(x)\right| \leq \frac{r_{k+1}}{2} \leq \frac{r}{2},
$$

and so

$$
\mathcal{H}^{1}\left(E_{N} \cap B(x, r) \cap \Gamma_{k^{\prime}, I^{\prime}}\right)=\mathcal{H}^{1}\left(B(x, r) \cap \Gamma_{k^{\prime}, I^{\prime}}\right) \gtrsim r .
$$

Hence, we may choose $\Sigma=\Gamma_{k^{\prime}, I^{\prime}}$.

Finally, for $1<r<\operatorname{diam}\left(E_{N}\right) \approx 1$, the condition (2.10) is satisfied with $\Sigma=$ $\Gamma_{0}$.

2.3. $\boldsymbol{E}_{N}$ has big conical energy. In this section we show that $E_{N}$ satisfies (2.1). We introduce additional notation. Analogously to the definition of $S_{k, I}$ for $k \in\{0,1,2,3\}$, for $I=\left(I^{\prime}, J\right) \in \mathcal{I}^{3} \times \mathcal{I}$ we define $S_{4, I}=G_{3, I^{\prime}}\left(S_{1, J}\right)$.

If $I \in \mathcal{I}^{k+j}$ is of the form $I=\left(I^{\prime}, I^{\prime \prime}\right) \in \mathcal{I}^{k} \times \mathcal{I}^{j}$, we will write

$$
S_{k, I}:=S_{k, I^{\prime}}, \quad \Gamma_{k, I}:=\Gamma_{k, I^{\prime}}, \quad G_{k, I}:=G_{k, I^{\prime}} .
$$

Lemma 2.7. Let $I=\left(I_{1}, I_{2}, I_{3}, I_{4}\right) \in \mathcal{I}^{4}$, and let $x \in S_{4, I} \subset \Gamma_{3, I} \subset E_{N}$. Then, for any $\theta \in[0, \pi)$ we have

$$
\int_{0}^{1} \frac{\mathcal{H}^{1}\left(K(x, \theta, \alpha, r) \cap E_{N}\right)}{r} \frac{d r}{r} \gtrsim N .
$$

Proof. Let $I \in \mathcal{I}^{4}, x \in S_{4, I}$ and $\theta \in[0, \pi)$ be as above. Recall that $L_{0}=$ $\{(x, 0): x \in \mathbb{R}\}, \rho$ is the counterclockwise rotation by $\pi / 4$, and $L_{k}=\rho^{k}\left(L_{0}\right) \in G(2,1)$. Observe that there exists some $k \in\{0,1,2,3\}$ such that $\theta-k \pi / 4 \in[0, \pi / 4)$. Fix such $k$. We are going to use $(\mathrm{P} 4)$ with respect to $\Gamma_{k, I}$ to arrive at (2.12).

Recall that $S_{k+1, I}=G_{k, I}\left(S_{1, I_{k+1}}\right)$, where $G_{k, I}=\tau \circ \rho^{k} \circ \delta^{k}$ for some translation $\tau$. Recall also that $G_{k, I}(\Gamma)=\Gamma_{k, I}$. Let $x^{\prime}=G_{k, I}^{-1}(x)$, and $\theta^{\prime}=\theta-k \pi / 4 \in[0, \pi / 4)$. Note that $V_{\theta^{\prime}}=\rho^{-k}\left(V_{\theta}\right)=G_{k, I}^{-1}\left(V_{\theta}\right)$. Using the fact that $G_{k, I}$ is a similarity with stretching factor $r_{k}$, we get

$$
\begin{aligned}
& \int_{0}^{1} \frac{\mathcal{H}^{1}\left(K(x, \theta, \alpha, r) \cap E_{N}\right)}{r} \frac{d r}{r} \geq \int_{0}^{1} \frac{\mathcal{H}^{1}\left(K(x, \theta, \alpha, r) \cap \Gamma_{k, I}\right)}{r} \frac{d r}{r} \\
& =\int_{0}^{1} r_{k} \frac{\mathcal{H}^{1}\left(K\left(x^{\prime}, \theta^{\prime}, \alpha, r_{k}^{-1} r\right) \cap \Gamma\right)}{r} \frac{d r}{r}=\int_{0}^{r_{k}^{-1}} \frac{\mathcal{H}^{1}\left(K\left(x^{\prime}, \theta^{\prime}, \alpha, s\right) \cap \Gamma\right)}{s} \frac{d s}{s} .
\end{aligned}
$$

Recall that $k$ was chosen in such a way that $\theta^{\prime} \in[0, \pi / 4)$. In order to use (P4), it only remains to show that $\operatorname{dist}\left(x^{\prime}, G\left(I^{\prime}\right)\right) \leq 2^{-N(M+1)}$ for some $I^{\prime} \in \mathcal{I}$.

Observe that $\gamma_{3, k}\left(S_{4, I}\right) \subset S_{k+1, I}$. We know from (2.11) that if $M \geq 6$, then

$$
\operatorname{dist}\left(x, S_{k+1, I}\right) \leq \operatorname{dist}\left(x, \gamma_{3, k}\left(S_{4, I}\right)\right) \leq\left|x-\gamma_{3, k}(x)\right| \leq r_{k+1}
$$


Thus,

$$
\begin{aligned}
\operatorname{dist}\left(x^{\prime}, S_{1, I_{k+1}}\right) & =\operatorname{dist}\left(G_{k, I}^{-1}(x), G_{k, I}^{-1}\left(S_{k+1, I}\right)\right)=r_{k}^{-1} \operatorname{dist}\left(x, S_{k+1, I}\right) \\
& \leq r_{k}^{-1} r_{k+1}=r_{1}=2^{-N(M+1)-1 / 2} \leq 2^{-N(M+1)} .
\end{aligned}
$$

$S_{1, I_{k+1}}$ was defined as $\sigma_{0}\left(I_{k+1}\right)=G\left(I_{k+1}\right)$, and so it follows from (P4) that the last term in (2.13) is greater than $C N$ for some absolute constant $C$. Thus, (2.12) holds.

Now we can finish the proof of Proposition 2.1. Observe that

$$
\begin{aligned}
\mathcal{H}^{1}\left(\bigcup_{I \in \mathcal{I}^{4}} S_{4, I}\right) & =\sum_{I^{\prime} \in \mathcal{I}^{3}, J \in \mathcal{I}} \mathcal{H}^{1}\left(G_{3, I^{\prime}}\left(S_{1, J}\right)\right) \\
& =(\# \mathcal{I})^{3} r_{3} \sum_{J \in \mathcal{I}} \mathcal{H}^{1}\left(S_{1, J}\right) \geq(\# \mathcal{I})^{3} r_{3} \sum_{J \in \mathcal{I}} \mathcal{H}^{1}(J) \\
& \stackrel{(P 3)}{=} 2^{-3 M} 2^{3 N(M+1)} 2^{-3 N(M+1)-3 / 2} 2^{-M+1}=2^{-4 M-1 / 2} \approx_{\alpha} 1,
\end{aligned}
$$

where we also used that $M$ is a constant depending only on $\alpha$. Together with Lemma 2.7, this shows that the set $E_{N}$ has the desired property (2.1), i.e.

$$
\begin{aligned}
& \int_{E_{N}} \int_{0}^{1} \frac{\mathcal{H}^{1}\left(K\left(x, \theta_{x}, \alpha, r\right) \cap E_{N}\right)}{r} \frac{d r}{r} d \mathcal{H}^{1}(x) \\
& \geq \sum_{I \in \mathcal{I} 4} \int_{S_{4, I}} \int_{0}^{1} \frac{\mathcal{H}^{1}\left(K\left(x, \theta_{x}, \alpha, r\right) \cap E_{N}\right)}{r} \frac{d r}{r} d \mathcal{H}^{1}(x) \gtrsim_{\alpha} N .
\end{aligned}
$$

Thus, the proof of Proposition 2.1 is complete. All that remains to prove is Lemma 2.2. We do that in the following two subsections.

2.4. Construction of $\boldsymbol{g}$. In this subsection we construct a function $g$ and a family of dyadic intervals $\mathcal{I}$ that satisfy (P1), (P2), and (P3). First, we define a family of auxiliary functions. For $j=1, \ldots, M$ we define $f_{j}:[-1,1] \rightarrow\left[-M^{-1} 2^{-j N}, M^{-1} 2^{-j N}\right]$ as

$$
f_{j}(t)=\frac{h\left(2^{j N} t\right)}{M 2^{j N}}
$$

where $h(t): \mathbb{R} \rightarrow[-1,1]$ is the 1-Lipschitz triangle wave:

$$
h(t)=|t \bmod 4-2|-1 .
$$

In the above $t \bmod 4$ denotes the unique number $s \in[0,4)$ such that $t=4 k+s$ for some $k \in \mathbb{Z}$.

Note that for all $j$ we have $\operatorname{Lip}\left(f_{j}\right)=M^{-1}$. For $j=1, \ldots, M$ we define also $g_{j}:[-1,1] \rightarrow\left[-M^{-1} 2^{-N+1}, M^{-1} 2^{-N+1}\right]$ as

$$
g_{j}(t)=\sum_{i=1}^{j} f_{i}(t),
$$

and we set $\Gamma_{j}=\operatorname{graph}\left(g_{j}\right) \subset B(0,1) \subset \mathbb{R}^{2}, g=g_{M}, \Gamma=\Gamma_{M}$. See Figure 2.2. Observe that $g$ is 1-Lipschitz.

Proof of (P1). We want to show that $g(1)=g(-1)=0$. Since $h$ is an even function, the functions $f_{j}$ and $g_{j}$ are also even. Hence, $g(1)=g(-1)$. Note also that if we have some function $\tilde{g}$ satisfying properties (P2) and (P4), then for any constant $C \in \mathbb{R}$ the function $\tilde{g}+C$ will also satisfy (P2) and (P4). In other words, these properties are invariant under adding constants. It follows that we can work with 
the function $g$ as defined above, prove (P2) and (P4), and at the end replace $g$ by $g-g(1)$. So the property (P1) is not an issue.
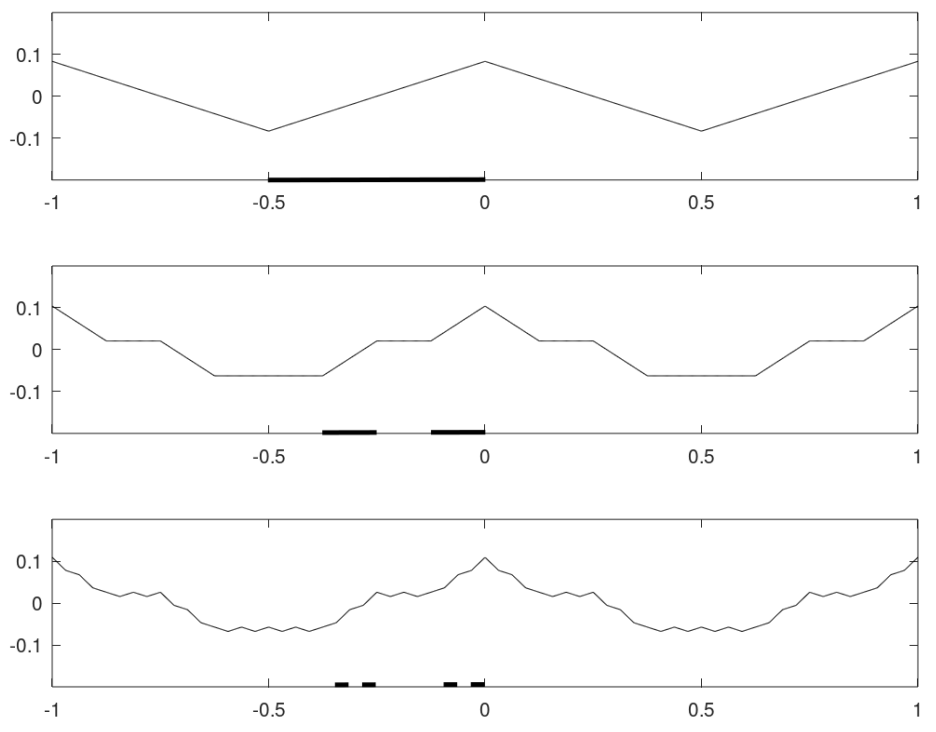

Figure 2.2. Top to bottom: graphs of $g_{1}, g_{2}$, and $g_{3}=g$ when $N=2$ and $M=3$. The thick segments denote intervals in $\mathcal{G}_{1}, \mathcal{G}_{2}$, and $\mathcal{G}_{3}$, respectively.

We proceed to define the family $\mathcal{I} \subset \Delta_{(M+1) N}$. Recall that $\Delta_{k}$ denotes the open dyadic intervals of length $2^{-k}$. Observe that for any $j$ the functions $f_{j}$ and $g_{j}$ are linear on each interval from $\Delta_{j N}$, and we have $f_{j}^{\prime}=M^{-1}$ on every second interval, and $f_{j}^{\prime}=-M^{-1}$ on the rest.

Set $\mathcal{G}_{j} \subset \Delta_{j N}$ to be the family of dyadic intervals $I$ contained in $[-1 / 2,1 / 2]$ such that for all $1 \leq i \leq j$ we have $f_{i}^{\prime}=M^{-1}$ on $I$. It is easy to see that each $\mathcal{G}_{j}$ consists of $2^{j N-j}$ disjoint intervals of length $2^{-j N}$, see Figure 2.2. We define also $\mathcal{I} \subset \Delta_{(M+1) N}$ as the family of dyadic intervals of length $2^{-(M+1) N}$ contained in $\bigcup_{I \in \mathcal{G}_{M}} I$.

Proof of (P3). By the definition above we have

$$
\# \mathcal{I}=2^{N} \cdot \# \mathcal{G}_{M}=2^{(M+1) N-M},
$$

so the property (P3) holds.

Proof of (P2). We have defined $\mathcal{G}_{j}$ in such a way that if $t \in I \in \mathcal{G}_{j}$ then $g_{j}^{\prime}(t)=j M^{-1}$. It follows that if $t \in I \in \mathcal{I}$, then $t \in J$ for some $J \in \mathcal{G}_{M}$, and so $g^{\prime}=1$. Thus, (P2) holds.

2.5. $\Gamma$ has big conical energy. This subsection is dedicated to proving (P4). We recall the statement for reader's convenience:

(P4) Let $\Gamma=\operatorname{graph}(g), G:[-1,1] \rightarrow \Gamma$ be the graph map $G(t)=(t, g(t))$. For any $I \in \mathcal{I}$, any $x \in \mathbb{R}^{2}$ with $\operatorname{dist}(x, G(I))<2^{-N(M+1)}$, and all $\theta \in[0, \pi / 4]$, we have

$$
\int_{0}^{1} \frac{\mathcal{H}^{1}(K(x, \theta, \alpha, r) \cap \Gamma)}{r} \frac{d r}{r} \gtrsim N
$$

Fix $x, I$, and $\theta$ as above. We will show (2.16). Since $\operatorname{dist}(x, G(I))<2^{-N(M+1)}$, there exists $t_{0} \in I$ such that $\left|x-G\left(t_{0}\right)\right| \leq 2^{-N(M+1)}$. Fix such $t_{0}$. 
For every $j=1, \ldots, M$ define $G_{j}(t)=\left(t, g_{j}(t)\right)$. For every $t \in \bigcup_{I \in \Delta_{j N}} I$ set $L_{j}(t) \subset \mathbb{R}^{2}$ to be the line tangent to $\Gamma_{j}$ at $G_{j}(t)$. We define also $I_{j}(t)$ as the unique interval from $\Delta_{j N}$ containing $t$. Note that, since $g_{j}$ is linear on intervals from $\Delta_{j N}$, we have $L_{j}(t)=L_{j}\left(t^{\prime}\right)$ whenever $t^{\prime} \in I_{j}(t)$. Denote by $L_{0}$ the $x$-axis. Thus,

Observe that if $I_{M}(t) \in \mathcal{G}_{M}$, then for each $1 \leq j \leq M$ we have $g_{j}^{\prime}(t)=j M^{-1}$.

$$
\measuredangle\left(L_{j}(t), L_{0}\right)=\arctan \left(j M^{-1}\right), \quad \text { and } \quad \measuredangle\left(L_{j}(t), V_{\pi / 4}\right) \leq \pi / 8 .
$$

Set $L_{j}=L_{j}(t)-\left(t, g_{j}(t)\right)$. Note that $(0,0) \in L_{j}$, and that the definition of $L_{j}$ does not depend on $t$, as long as $I_{M}(t) \in \mathcal{G}_{M}$. Since $\theta \in[0, \pi / 4]$, it follows from (2.17) that there exists some $1 \leq j \leq M$ such that

$$
\begin{aligned}
\measuredangle\left(V_{\theta}, L_{j}\right) & \leq \max _{1 \leq i \leq M}\left(\arctan \left(i M^{-1}\right)-\arctan \left((i-1) M^{-1}\right)\right) \\
& =\arctan \left(M^{-1}\right) \leq M^{-1} .
\end{aligned}
$$

Fix such $j$. Recall that $M=100\left\lceil\alpha^{-1}\right\rceil$, and so

$$
\measuredangle\left(V_{\theta}, L_{j}\right) \leq M^{-1} \leq \frac{\alpha}{10} \text {. }
$$

Hence, for any $r>0$

$$
K(x, \theta, \alpha, r) \supset K\left(x, L_{j}, \alpha / 2, r\right) .
$$

Lemma 2.8. For $t \in[-1,1]$ we have

$$
\left|G(t)-G_{j}(t)\right|=\left|g(t)-g_{j}(t)\right| \leq 2 M^{-1} 2^{-N(j+1)} .
$$

Proof. The estimate follows immediately from the definition of $g$ and $g_{j}$ :

$$
\left|g(t)-g_{j}(t)\right|=\left|\sum_{i=j+1}^{M} f_{i}(t)\right| \leq \sum_{i=j+1}^{M}\left|f_{i}(t)\right| \leq \sum_{i=j+1}^{\infty} \frac{1}{M} 2^{-i N} \leq 2 M^{-1} 2^{-N(j+1)} .
$$

Recall that $t_{0} \in I \in \mathcal{I}$ was such that $\left|x-G\left(t_{0}\right)\right| \leq 2^{-N(M+1)}$. Set $x^{\prime}=G_{j}\left(t_{0}\right)$. Then, by the lemma above, we have

$$
\left|x-x^{\prime}\right| \leq\left|x-G\left(t_{0}\right)\right|+\left|G\left(t_{0}\right)-G_{j}\left(t_{0}\right)\right| \leq 2^{-N(M+1)}+2^{-N(j+1)} \leq 2^{-N(j+1)+1} .
$$

Let $I^{\prime} \in \mathcal{G}_{j}$ be the unique dyadic interval in $\Delta_{j N}$ containing $I$. That is, $I^{\prime}=I_{j}\left(t_{0}\right)$.

Let $K(x, V, \alpha, r, R)$ denote the twice truncated cone $K(x, V, \alpha, R) \backslash B(x, r)$. In the lemma below we show that for all the scales between $2^{-N(j+1)}$ and $2^{-N j}, G\left(I^{\prime}\right)$ has large intersection with the the twice truncated cone centered at $x^{\prime}$ with direction $L_{j}$ corresponding to that scale.

Lemma 2.9. For $t \in I^{\prime}$ such that $\left|G(t)-x^{\prime}\right| \geq 2^{-N(j+1)}$ we have $G(t) \in$ $K\left(x^{\prime}, L_{j}, \alpha / 8\right)$. Moreover, for integers $k$ satisfying $N j \leq k \leq N(j+1)-1$ we have

$$
\mathcal{H}^{1}\left(G\left(I^{\prime}\right) \cap K\left(x^{\prime}, L_{j}, \alpha / 8,2^{-k-1}, 2^{-k+2}\right)\right) \gtrsim 2^{-k} .
$$

Proof. Let $t \in I^{\prime}$ satisfy $\left|G(t)-x^{\prime}\right| \geq 2^{-N(j+1)}$. Recall that, since $I^{\prime} \in \mathcal{G}_{j}$, the set $G_{j}\left(I^{\prime}\right)$ is a segment parallel to $L_{j}$. We also know that $x^{\prime}=G_{j}\left(t_{0}\right) \in G_{j}\left(I^{\prime}\right)$, and so by Lemma 2.8

$$
\operatorname{dist}\left(G(t), L_{j}+x^{\prime}\right) \leq\left|G(t)-G_{j}(t)\right| \leq 2 M^{-1} 2^{-N(j+1)} \leq \sin (\alpha / 8)\left|G(t)-x^{\prime}\right|,
$$

where we also used that $M=100\left\lceil\alpha^{-1}\right\rceil$ and we assume $\alpha$ to be so small that $\alpha / 8 \leq$ $2 \sin (\alpha / 8)$. Thus, $G(t) \in K\left(x^{\prime}, L_{j}, \alpha / 8\right)$. 
Now, let $k$ be an integer such that $N j \leq k \leq N(j+1)-1$. Let $t \in I^{\prime}$ be such that $2^{-k}<\left|t-t_{0}\right|$, so that

$$
\begin{aligned}
\left|G(t)-x^{\prime}\right| & \geq\left|G_{j}(t)-G_{j}\left(t_{0}\right)\right|-\left|G(t)-G_{j}(t)\right| \geq\left|t-t_{0}\right|-2 M^{-1} 2^{-N(j+1)} \\
& \geq 2^{-k}-2 M^{-1} 2^{-N(j+1)} \geq 2^{-N(j+1)} .
\end{aligned}
$$

Hence, by our previous result, $G(t) \in K\left(x^{\prime}, L_{j}, \alpha / 8\right)$. At the same time, the calculation above shows that $\left|G(t)-x^{\prime}\right| \geq 2^{-k-1}$. Similarly,

$$
\left|G(t)-x^{\prime}\right| \leq\left|G_{j}(t)-G_{j}\left(t_{0}\right)\right|+\left|G(t)-G_{j}(t)\right| \leq \sqrt{2}\left|t-t_{0}\right|+2 M^{-1} 2^{-N(j+1)} .
$$

Hence, for $t \in I^{\prime}$ such that $2^{-k} \leq\left|t-t_{0}\right| \leq 2^{-k+1}$ we have

$$
2^{-k-1} \leq\left|G(t)-x^{\prime}\right| \leq 2^{-k+2} \text {. }
$$

That is, for $t \in I^{\prime}$ with $2^{-k} \leq\left|t-t_{0}\right| \leq 2^{-k+1}$ we have

$$
G(t) \in K\left(x^{\prime}, L_{j}, \alpha / 8,2^{-k-1}, 2^{-k+2}\right) .
$$

Since $G$ is bilipschitz, (2.22) follows.

Later on we will need the following simple lemma about the inclusions of twice truncated cones.

Lemma 2.10. Let $x_{1}, x_{2} \in \mathbb{R}^{2}, L \in G(2,1), r>0$ and $\alpha_{0} \in(0, \pi / 50)$. Suppose that $\left|x_{1}-x_{2}\right| \leq \sin \left(\alpha_{0}\right) r$. Then

$$
K\left(x_{1}, L, \alpha_{0}, \sin \left(\alpha_{0}\right)^{-1}\left|x_{1}-x_{2}\right|, r\right) \subset K\left(x_{2}, L, 8 \alpha_{0}, 2 r\right) .
$$

Proof. Let $y \in K\left(x_{1}, L, \alpha_{0}, \sin \left(\alpha_{0}\right)^{-1}\left|x_{1}-x_{2}\right|, r\right)$, so that $\sin \left(\alpha_{0}\right)^{-1}\left|x_{1}-x_{2}\right|<$ $\left|y-x_{1}\right| \leq r$ and $\operatorname{dist}\left(y, L+x_{1}\right) \leq \sin \left(\alpha_{0}\right)\left|y-x_{1}\right|$. It is clear that for any $p \in L+x_{1}$ we have $\operatorname{dist}\left(p, L+x_{2}\right)=\left|x_{1}-x_{2}\right|$, and so

$$
\begin{aligned}
\operatorname{dist}\left(y, L+x_{2}\right) & \leq \operatorname{dist}\left(y, L+x_{1}\right)+\left|x_{1}-x_{2}\right| \leq \sin \left(\alpha_{0}\right)\left|y-x_{1}\right|+\sin \left(\alpha_{0}\right)\left|y-x_{1}\right| \\
& \leq 2 \sin \left(\alpha_{0}\right)\left|y-x_{2}\right|+2 \sin \left(\alpha_{0}\right)\left|x_{1}-x_{2}\right| .
\end{aligned}
$$

It is easy to check that for $\alpha_{0} \in(0, \pi / 50)$ we have $4 \sin \left(\alpha_{0}\right) \leq \sin \left(8 \alpha_{0}\right)$, and so

$$
\operatorname{dist}\left(y, L+x_{2}\right) \leq \frac{\sin \left(8 \alpha_{0}\right)}{2}\left(\left|y-x_{2}\right|+\left|x_{1}-x_{2}\right|\right) .
$$

At the same time, we have

$$
\left|y-x_{2}\right| \geq\left|y-x_{1}\right|-\left|x_{1}-x_{2}\right| \geq\left(\sin \left(\alpha_{0}\right)^{-1}-1\right)\left|x_{1}-x_{2}\right| \geq\left|x_{1}-x_{2}\right| .
$$

Putting the two estimates together gives $y \in K\left(x_{2}, L, 8 \alpha_{0}\right)$. To see that $y \in B\left(x_{2}, 2 r\right)$, note that $\left|y-x_{2}\right| \leq\left|y-x_{1}\right|+\left|x_{1}-x_{2}\right| \leq 2 r$.

Recall that in (2.22) we showed a lower bound on the length of intersection of $G\left(I^{\prime}\right)$ with a cone centered at $x^{\prime}$. However, to prove (2.16) we need information about the intersections with cones centered at $x$. We use (2.22) and Lemma 2.10 to get the following.

Lemma 2.11. Let $k$ be an integer such that $\alpha^{-1} 2^{-N(j+1)+9}<2^{-k} \leq 2^{-N j-3}$. Then, we have

$$
\mathcal{H}^{1}\left(G\left(I^{\prime}\right) \cap K\left(x, L_{j}, \alpha / 2,2^{-k}\right)\right) \gtrsim 2^{-k},
$$

Proof. First, recall that $x^{\prime}=G_{j}\left(t_{0}\right)$ and $\left|x-x^{\prime}\right| \leq 2^{-N(j+1)+1}$ by $(2.21)$. Using our assumptions on $k$, and that we assume $\alpha$ to be so small that $\sin (\alpha / 8) \geq \alpha / 16$, we get

$$
\sin (\alpha / 8)^{-1}\left|x-x^{\prime}\right| \leq \alpha^{-1} 2^{-N(j+1)+5} \leq 2^{-k-4}<2^{-k-1}
$$


Hence, we may apply Lemma 2.10 with $x_{1}=x^{\prime}, x_{2}=x, L=L_{j}, \alpha_{0}=\alpha / 8, r=$ $2^{-k-1}$, to get

$$
K\left(x^{\prime}, L_{j}, \alpha / 8, \sin (\alpha / 8)^{-1}\left|x-x^{\prime}\right|, 2^{-k-1}\right) \subset K\left(x, L_{j}, \alpha / 2,2^{-k}\right) .
$$

Since $\sin (\alpha / 8)^{-1}\left|x-x^{\prime}\right| \leq 2^{-k-4}$ by (2.24), it follows from the above that

$$
K\left(x^{\prime}, L_{j}, \alpha / 8,2^{-k-4}, 2^{-k-1}\right) \subset K\left(x, L_{j}, \alpha / 2,2^{-k}\right) .
$$

Note that we have $N j \leq k-3 \leq N(j+1)-1$ due to our assumptions on $k$. Thus, we may use (2.22) to get

$$
\mathcal{H}^{1}\left(G\left(I^{\prime}\right) \cap K\left(x, L_{j}, \alpha / 8,2^{-k-4}, 2^{-k-1}\right)\right) \gtrsim 2^{-k} .
$$

Together with (2.25), this concludes the proof.

We are ready to finish the proof of Lemma 2.2.

Proof of (P4). We want to show that

$$
\int_{0}^{1} \frac{\mathcal{H}^{1}(K(x, \theta, \alpha, r) \cap \Gamma)}{r} \frac{d r}{r} \gtrsim N .
$$

We use (2.20) to write

$$
\begin{aligned}
\int_{0}^{1} \frac{\mathcal{H}^{1}(K(x, \theta, \alpha, r) \cap \Gamma)}{r} \frac{d r}{r} & \geq \int_{0}^{1} \frac{\mathcal{H}^{1}\left(K\left(x, L_{j}, \alpha / 2, r\right) \cap \Gamma\right)}{r} \frac{d r}{r} \\
& \geq \int_{\alpha^{-1} 2^{-N(j+1)+10}}^{2^{-N j-3}} \frac{\mathcal{H}^{1}\left(K\left(x, L_{j}, \alpha / 2, r\right) \cap \Gamma\right)}{r} \frac{d r}{r}
\end{aligned}
$$

Note that $\alpha^{-1} 2^{-N(j+1)+10}<2^{-N j-3}$ due to the assumption $N \geq 100\left(1+\log _{2}\left(\alpha^{-1}\right)\right)$. Now let $\alpha^{-1} 2^{-N(j+1)+10} \leq r<2^{-N j-3}$, and let $k$ be the unique integer such that $2^{-k} \leq r<2^{-k+1}$. Then, $k$ satisfies the assumptions of Lemma 2.11, and we get

$$
\mathcal{H}^{1}\left(K\left(x, L_{j}, \alpha / 2, r\right) \cap \Gamma\right) \geq \mathcal{H}^{1}\left(K\left(x, L_{j}, \alpha / 2,2^{-k}\right) \cap \Gamma\right) \gtrsim 2^{-k} \approx r .
$$

It follows from (2.27) and the above that

$$
\begin{aligned}
& \int_{0}^{1} \frac{\mathcal{H}^{1}(K(x, V, \alpha, r) \cap \Gamma)}{r} \frac{d r}{r} \gtrsim \int_{\alpha^{-1} 2^{-N(j+1)+10}}^{2^{-N j-3}} 1 \frac{d r}{r} \\
& =\log (2)\left(N(j+1)-10-\log _{2}\left(\alpha^{-1}\right)-N j-3\right) \\
& =\log (2)\left(N-\log _{2}\left(\alpha^{-1}\right)-13\right) \geq \frac{N}{100},
\end{aligned}
$$

where we used the assumption $N \geq 100\left(1+\log _{2}\left(\alpha^{-1}\right)\right)$ in the last inequality. Thus, the proof of (2.26) is finished.

\section{Example of Joyce and Mörters}

In this section we will show that the measure $\mu$ constructed in [JM00] satisfies $\operatorname{BPBE}(2)$, but does not satisfy $\operatorname{BPBE}(1)$. Hence, Theorem 1.10 is a true improvement on its $\mathcal{E}_{\mu, 1}$ analogue [CT20, Theorem 10.2].

3.1. Construction of $\boldsymbol{\mu}$. For reader's convenience, we sketch out the construction of Joyce and Mörters below. 
Let $M \geq 3$ be a large constant, and $1 / 2<\beta_{k}<1$ be a sequence of numbers converging to 1 . For $k \geq 1$ we define $m_{k}=M k, m(k)=m_{1} \ldots m_{k}=M^{k} k$ !, and

$$
\sigma_{k}=\left(\frac{k+1}{k}\right)^{\beta_{k}} \text {. }
$$

We set also $\alpha_{j}=2^{-n} \pi$ for all $2^{n} \leq j<2^{n+1}, n \geq 0$.

We proceed to define a compact set $E \subset \mathbb{R}^{2}$ on which the measure $\mu$ will be supported. First, let $E_{0}$ be a closed ball of diameter 1 . We place $m_{1}$ closed balls of diameter $2 r_{1}:=\sigma_{1} / m_{1}$ inside $E_{0}$. We do it in such a way, that

- their centers lie on the diameter of $E_{0}$ forming angle $\alpha_{1}$ with the $x$ axis,

- the boundaries of the first and the last ball touch the boundary of $E_{0}$,

- they overlap as little as possible, i.e. the distance between the centers of two neighbouring balls is $\left(1-\sigma_{1} / m_{1}\right) /\left(m_{1}-1\right)$.

We call these balls the balls of generation 1 , we denote their family by $\mathcal{B}_{1}$, and we set $E_{1}=\bigcup_{B \in \mathcal{B}_{1}} B$.

Now suppose that $E_{k}$ has already been defined as a union of balls $\bigcup_{B \in \mathcal{B}_{k}} B$, and that $\# \mathcal{B}_{k}=m(k)$. Inside every ball $B \in \mathcal{B}_{k}$ we place $m_{k+1}$ closed balls of diameter $2 r_{k+1}:=\sigma_{1} \ldots \sigma_{k+1} / m(k+1)$. We do it in such a way, that

- their centers lie on the diameter of $B$ forming angle $\sum_{i=1}^{k+1} \alpha_{i}$ with the $x$ axis,

- the boundaries of the first and the last ball touch touch the boundary of $B$,

- they overlap as little as possible, i.e. the distance between the centers of two neighbouring balls is

$$
d_{k+1}:=\frac{\sigma_{1} \ldots \sigma_{k}}{m(k)} \cdot \frac{1-\sigma_{k+1} / m_{k+1}}{m_{k+1}-1} .
$$

The balls defined above are called the balls of generation $(k+1)$, and their family is denoted by $\mathcal{B}_{k+1}$. Clearly, $\# \mathcal{B}_{k+1}=m_{k+1} \cdot m(k)=m(k+1)$. We set $E_{k+1}=$ $\bigcup_{B \in \mathcal{B}_{k+1}} B$, and $E=\bigcap_{k \geq 0} E_{k}$.

It is shown in [JM00, §2.1] that if $M$ is chosen appropriately, then two balls of generation $(k+1)$ may intersect only if they are contained in the same ball of generation $k$. It follows that there exists a natural probability measure $\mu$ supported on $E$ defined by

$$
\mu(B)=m(k)^{-1} \quad \text { for } B \in \mathcal{B}_{k}, k \geq 1 .
$$

If the sequence $\beta_{k}$ is chosen properly, the set $E$ has the following curious property: it is of non- $\sigma$-finite length, but all the projections of $E$ onto lines are of zero length. Moreover, the Menger curvature of $E$ is finite. However, we will not use those properties.

3.2. $\mathrm{BPBE}(2)$ holds. In [JM00, §2.1] Joyce and Mörters construct a function $\varphi:\left[0, d_{1}\right) \rightarrow \mathbb{R}$ satisfying $\varphi(r)<r$ and

$$
\int_{0}^{d_{1}} \frac{\varphi(r)^{2}}{r^{3}} d r<\infty .
$$

They also show that for $0<r<d_{1}$ the measure $\mu$ satisfies $\mu(B(x, r)) \leq 84 \varphi(r)$. It follows easily that $\mu(B(x, r)) \leq C_{1} r$ for $C_{1}=\max \left(84,1 / d_{1}\right)$ and all $r>0$, and so $\mu$ satisfies (1.3). Furthermore, by the observations above and the fact that $\mu\left(\mathbb{R}^{2}\right)=1$, 
for all $x \in E=\operatorname{supp} \mu$ we have

$$
\int_{0}^{\infty}\left(\frac{\mu(B(x, r))}{r}\right)^{2} \frac{d r}{r} \lesssim \int_{0}^{d_{1}} \frac{\varphi(r)^{2}}{r^{3}} d r+\int_{d_{1}}^{\infty} \frac{1}{r^{3}} d r \leq M_{0}
$$

for some $M_{0}$ depending only on $d_{1}$ and $\varphi$.

Obviously, for any $\theta \in[0, \pi / 2), \alpha \in(0,1), R>0$, we have

$$
\mathcal{E}_{\mu, 2}(x, \theta, \alpha, R)=\int_{0}^{R}\left(\frac{\mu(K(x, \theta, \alpha, r))}{r}\right)^{2} \frac{d r}{r} \leq \int_{0}^{\infty}\left(\frac{\mu(B(x, r))}{r}\right)^{2} \frac{d r}{r},
$$

and so the $\operatorname{BPBE}(2)$ condition is trivially satisfied.

Let us note that the boundedness of nice singular integral operators on $L^{2}(\mu)$ for this particular measure $\mu$ is not a new result. It is well known that measures satisfying (3.2) behave well with respect to SIOs. For example, one can use (3.2) and [Mat96, Theorem 2.2] to prove local curvature condition for $\mu$, and then boundedness of Cauchy transform follows from [Tol99, Theorem 1.1].

3.3. $\mathcal{E}_{\boldsymbol{\mu}, \mathbf{1}}$ is not bounded. Let $x \in E, \theta \in[0, \pi / 2)$, and $\alpha \in(0, \pi / 100)$ be given. We will show that

$$
\mathcal{E}_{\mu, 1}(x, \theta, \alpha, 1)=\int_{0}^{1} \frac{\mu(K(x, \theta, \alpha, r))}{r} \frac{d r}{r}=\infty .
$$

Definition 3.1. We will say that an integer $k$ is a good index if

$$
\left|\left(\sum_{j=1}^{k} \alpha_{j}-N \pi\right)-\theta\right| \leq \frac{\alpha}{16}
$$

where $N$ is the integer satisfying $2^{N} \leq k<2^{N+1}$. By the definition of $\alpha_{j}$, this is equivalent to

$$
\left|\left(k-2^{N}+1\right) \frac{\pi}{2^{N}}-\theta\right| \leq \frac{\alpha}{16} .
$$

Our strategy is the following: first, we show that there are many good indices. Then, we prove that if $k$ is a good index, then $\mu\left(K\left(x, \theta, \alpha, 2 r_{k}\right)\right) r_{k}^{-1}$ is large. Put together, the two facts will imply (3.3).

We define $N_{0}=N_{0}(\alpha)$ to be a large integer, to be fixed in Lemmas 3.2 and 3.3.

Lemma 3.2. If $N_{0}=N_{0}(\alpha)$ is large enough, then for all $N>N_{0}$ we have a large portion of good indices satisfying $2^{N} \leq k<2^{N+1}$, that is,

$$
\#\left\{2^{N} \leq k<2^{N+1}: k \text { is a good index }\right\} \gtrsim 2^{N} \alpha .
$$

Proof. Let $N_{0}$ be so big that $2^{-N_{0}} \pi<\alpha / 100$, and let $N>N_{0}$. Let $2^{N} \leq k_{0}<$ $2^{N+1}$ be the index minimizing $\left|\left(k_{0}-2^{N}+1\right) \pi 2^{-N}-\theta\right|$. It is clear that

$$
\left|\left(k_{0}-2^{N}+1\right) 2^{-N} \pi-\theta\right| \leq 2^{-N} \pi,
$$

and so it follows from (3.5) that all integers $k$ such that $2^{N} \leq k<2^{N+1}$ and $\mid(k-$ $\left.k_{0}\right) 2^{-N} \pi \mid \leq \alpha / 50$ are good indices. It is easy to see that there are at least $C 2^{N} \alpha$ such integers, where $C$ is some absolute constant.

Recall that $r_{k}$ was the radius of balls of $k$-th generation, and $x \in E$ is arbitrary. For $k \geq 1$ let $B_{k} \in \mathcal{B}_{k}$ be a ball of generation $k$ containing $x$ (there may be two such balls, in which case we just choose one). 
Lemma 3.3. If $N_{0}=N_{0}(\alpha)$ is large enough, then for all good indices $k \geq 2^{N_{0}}$ we have

$$
\mu\left(K\left(x, \theta, \alpha, 2 r_{k}\right)\right) \gtrsim \mu\left(B_{k}\right) .
$$

Proof. Let $y$ be the center of $B_{k+1}$, so that $|x-y| \leq r_{k+1}$. By construction,

$$
r_{k+1}=r_{k} \sigma_{k+1}(M k)^{-1} \leq r_{k} k^{-1} .
$$

Since $k \geq 2^{N_{0}}$, for $N_{0}$ big enough we get

$$
|x-y| \leq r_{k+1} \leq \frac{\sin (\alpha / 50) r_{k}}{2}
$$

Then, it follows from Lemma 2.10 that

$$
K\left(y, \theta, \alpha / 8, \sin (\alpha / 8)^{-1}|x-y|, r_{k}\right) \subset K\left(x, \theta, \alpha, 2 r_{k}\right) .
$$

Since $\sin (\alpha / 8)^{-1}|x-y| \leq r_{k} / 2$ by (3.7), we get

$$
K\left(y, \theta, \alpha / 8, r_{k} / 2, r_{k}\right) \subset K\left(x, \theta, \alpha, 2 r_{k}\right) .
$$

On the other hand, using the definition of good index (3.4) we arrive at

$$
K\left(y, \sum_{j=1}^{k} \alpha_{j}-N \pi, \alpha / 50, r_{k} / 2, r_{k}\right) \subset K\left(y, \theta, \alpha / 8, r_{k} / 2, r_{k}\right) .
$$

For brevity, set $\mathrm{K}$ to be the cone from the left hand side above, and let $L$ be the axis of K. Recall that the diameter of $B_{k}$ (let us call it $D$ ) forms angle $\sum_{j=1}^{k} \alpha_{j}-N \pi$ with the $x$ axis; that is, $D$ is parallel to $L$. Since $y$ is the center of $B_{k+1}$, it follows from the construction of $E$ that $y \in D$. Hence, $D \subset L$.

We claim that the balls of generation $(k+1)$ contained in $B_{k} \cap B\left(y, r_{k}\right) \backslash B\left(y, r_{k} / 2\right)$, are in fact contained in $\mathrm{K}$. Indeed, suppose $z$ belongs to such ball, so that

$$
\operatorname{dist}(z, L)=\operatorname{dist}(z, D) \leq r_{k+1} \stackrel{(3.7)}{\leq} \frac{\sin (\alpha / 50) r_{k}}{2} r_{k} \leq \sin (\alpha / 50)|z-y|
$$

Thus, $z \in \mathrm{K}$.

Since $y \in D$ and $B_{k}$ is a ball of radius $r_{k}$, it follows that a large portion of balls of generation $(k+1)$ contained in $B_{k}$ is also contained in $B\left(y, r_{k}\right) \backslash B\left(y, r_{k} / 2\right)$. That is, they are of the type considered above. Hence,

$$
\mu(\mathrm{K}) \gtrsim \mu\left(B_{k}\right)
$$

By (3.9) and (3.8) we have $\mathrm{K} \subset K\left(x, \theta, \alpha, 2 r_{k}\right)$, and so the proof is finished.

Lemma 3.4. For $k \geq 2$

$$
\frac{\mu\left(B_{k}\right)}{2 r_{k}} \gtrsim \frac{1}{k}
$$

Proof. By the definition of $\mu(3.1), r_{k}$, and $\sigma_{k}$ we have

$$
\begin{aligned}
\frac{\mu\left(B_{k}\right)}{2 r_{k}} & =m(k)^{-1} \frac{m(k)}{\sigma_{1} \ldots \sigma_{k}}=\frac{1}{\sigma_{1} \ldots \sigma_{k}}=\left(\frac{1}{2}\right)^{\beta_{1}} \ldots\left(\frac{k}{k+1}\right)^{\beta_{k}} \\
& \geq \frac{1}{2} \ldots \frac{k}{k+1}=\frac{1}{k+1},
\end{aligned}
$$

where in the last inequality we used the fact that $1 / 2<\beta_{k}<1$.

We are ready to finish the proof of the estimate (3.3). 
Proof of (3.3). Observe that if $k>N_{0}$ is a good index, then by Lemma 3.3 and Lemma 3.4 for $r \in\left(2 r_{k}, 4 r_{k}\right)$

$$
\frac{\mu(K(x, \theta, \alpha, r))}{r} \gtrsim \frac{1}{k}
$$

and so

$$
\int_{2 r_{k}}^{4 r_{k}} \frac{\mu(K(x, \theta, \alpha, r))}{r} \frac{d r}{r} \gtrsim \frac{1}{k} .
$$

Recall that $r_{k+1} \leq k^{-1} r_{k}$ by (3.6). Hence,

$$
\begin{aligned}
\int_{0}^{1} \frac{\mu(K(x, \theta, \alpha, r))}{r} \frac{d r}{r} & \geq \sum_{k \geq 2^{N_{0}}} \int_{2^{r_{k}}}^{4 r_{k}} \frac{\mu(K(x, \theta, \alpha, r))}{r} \frac{d r}{r} \\
& \geq \sum_{N=N_{0}}^{\infty} \sum_{\substack{2^{N} \leq k<2^{N+1} \\
k \text { is good }}} \int_{2 r_{k}}^{4 r_{k}} \frac{\mu(K(x, \theta, \alpha, r))}{r} \frac{d r}{r} \\
& \gtrsim \sum_{N=N_{0}}^{\infty} \sum_{\substack{2^{N} \leq k<2^{N+1} \\
k \text { is good }}} \frac{1}{k} \sum_{\substack{\text { (3.10) } \\
2^{N} \leq k<2^{N+1} \\
k \text { is good }}}^{\infty} 2^{-N} \sum_{N=N_{0}}^{\infty e m m a} 2^{-N} 2^{N} \alpha=\infty
\end{aligned}
$$

\section{References}

[AT15] AzzAM, J., and X. Tolsa: Characterization of $n$-rectifiability in terms of Jones' square function: Part II. - Geom. Funct. Anal. 25:5, 2015, 1371-1412.

[BN21] BAdger, M., and L. NAPLes: Radon measures and Lipschitz graphs. - Bull. Lond. Math. Soc., 2021.

[CT20] Chang, A., and X. Tolsa: Analytic capacity and projections. - J. Eur. Math. Soc. (JEMS) 22:12, 2020, 4121-4159.

[Dąb21] Dąbrowski, D.: Cones, rectifiability, and singular integral operators. - Rev. Mat. Iberoam., 2021, DOI 10.4171/RMI/1301.

[Dav91] David, G.: Wavelets and singular integrals on curves and surfaces. - Lecture Notes in Math. 1465, Springer, 1991.

[DS91] DAvid, G., and S. Semmes: Singular integrals and rectifiable sets in $\mathbb{R}^{n}$ : Au-delà des graphes lipschitziens. - Astérisque 193, 1991.

[DS93a] David, G., and S. Semmes: Analysis of and on uniformly rectifiable sets. - Math. Surveys Monogr. 38, Amer. Math. Soc., Providence, RI, 1993.

[DS93b] DAvid, G., and S. Semmes: Quantitative rectifiability and Lipschitz mappings. - Trans. Amer. Math. Soc. 337:2, 1993, 855-889.

[GS19] Girela-SARRIón, D.: Geometric conditions for the $L^{2}$-boundedness of singular integral operators with odd kernels with respect to measures with polynomial growth in $\mathbb{R}^{d}$. - J. Anal. Math. 137:1, 2019, 339-372.

[JM00] JoyCE, H., and P. MÖRTERs: A set with finite curvature and projections of zero length. - J. Math. Anal. Appl. 247:1, 2000, 126-135.

[MO18] Martikainen, H., and T. Orponen: Characterising the big pieces of Lipschitz graphs property using projections. - J. Eur. Math. Soc. (JEMS) 20:5, 2018, 1055-1073. 
[Mat96] Mattila, P.: On the analytic capacity and curvature of some Cantor sets with non- $\sigma$ finite length. - Publ. Mat. 40:1, 1996, 195-204.

[Orp21] Orponen, T.: Plenty of big projections imply big pieces of Lipschitz graphs. - Invent. Math. 226:2, 2021, 653-709.

[Tol99] Tolsa, X.: $L^{2}$-boundedness of the Cauchy integral operator for continuous measures. Duke Math. J. 98:2, 1999, 269-304.

[Tol09] Tolsa, X.: Uniform rectifiability, Calderón-Zygmund operators with odd kernel, and quasiorthogonality. - Proc. Lond. Math. Soc. (3) 98:2, 2009, 393-426.

[Tol12] TolsA, X.: Mass transport and uniform rectifiability. - Geom. Funct. Anal. 22:2, 2012, $478-527$.

Received 30 November 2020 • Accepted 8 April 2021 • Published online 4 January 2022

Damian Dąbrowski

Universitat Autònoma de Barcelona, Departament de Matemàtiques

and Barcelona Graduate School of Mathematics (BGSMath)

Edifici C Facultat de Ciències

08193 Bellaterra, Barcelona, Catalonia, Spain

and University of Jyväskylä, Department of Mathematics and Statistics

P.O. Box 35 (MaD), 40014 University of Jyväskylä, Finland

damian.m.dabrowski@jyu.fi 